\title{
Prevalência e fatores associados ao consumo abusivo e à dependência de álcool
}

\author{
Prevalence and associated factors of alcohol abuse \\ and alcohol addiction
}

Luciano Nery Ferreira ${ }^{1}$

José Patrício Bispo Júnior ${ }^{2}$

Zenilda Nogueira Sales ${ }^{1}$

Cezar Augusto Casotti ${ }^{1}$

Antonio Carlos Ricardo Braga Junior ${ }^{2}$

\footnotetext{
${ }^{1}$ Departamento de Saúde, Universidade Estadual do Sudoeste da Bahia. Av. José Moreira Sobrinho $\mathrm{s} / \mathrm{n}^{\circ}$, Jequiezinho. 45.206-190 Jequié BA Brasil. lnery@uesb.edu.br ${ }^{2}$ Instituto Multidisciplinar em Saúde, Universidade Federal da Bahia.
}

\begin{abstract}
The scope of this study is to estimate the prevalence of alcohol abuse and alcohol addiction and the respective associated factors in the urban population of a city of northeastern Brazil. It is a population-based cross-sectional study that investigated the consumption of alcohol of 270 people living in the urban area of Jequié, State of Bahia. Alcohol abuse, defined by a score of $\geq 8$ from responses to the Alcohol Use Disorders Identification Test (AUDIT). Alcohol addiction was identified by a score $\geq 2$ obtained in the application of the Cut down, Annoyed by criticism, Guilty and Eye-opener (CAGE). The analysis used the logistic regression model. The prevalence of alcohol abuse was $18.5 \%$. For alcohol addiction it was 10.4\%. After adjusted analysis, the groups with greater alcohol abuse were men $(O R=5.56)$ and young $(O R=5.41)$. Professing evangelical belief was inversely associated with alcohol abuse (OR $=0.04$ ). An association between alcohol abuse was established in the young, males, and smoking, whereas the inverse association was observed with those professing evangelical religious beliefs.
\end{abstract}

Key words Alcohol drinking, Alcoholism, Socioeconomic factors, Cross-sectional studies
Resumo O objetivo deste estudo é estimar a prevalência do consumo abusivo e da dependência de bebidas alcoólicas e os respectivos fatores associados em população urbana de um município do interior do Nordeste brasileiro. Estudo transversal de base populacional que investigou o consumo de bebidas alcoólicas em 270 indivíduos residentes na zona urbana de Jequié (BA). O uso abusivo de álcool, definido por uma pontuação $\geq 8 a$ partir das respostas ao Alcohol Use Disorders Identification Test (AUDIT). A dependência de álcool foi identificada por pontuação $\geq 2$ obtida na aplicação do Cut-down, Annoyed by criticism, Guilty e Eye-opener (CAGE). Análise utilizou o modelo de regressão logística. A prevalência do uso abusivo de bebidas alcoólicas foi de 18,5. Para a dependência de álcool, 10,4\%. Após análise ajustada, os grupos que apresentaram maior uso abusivo de bebidas alcoólicas foram os homens $(O R=$ 5,56) e os jovens $(O R=5,41)$. Professar crença evangélica apresentou associação inversa com o uso abusivo de álcool (OR =0,04). Estabeleceu-se associação entre o uso abusivo de bebidas alcoólicas e a faixa etária jovem, gênero masculino, e tabagismo, ao passo que associação inversa deste desfecho com crença religiosa evangélica.

Palavras-chave Consumo de bebidas alcoólicas, Alcoolismo, Fatores Socioeconômicos, Estudos transversais 


\section{Introdução}

O consumo de álcool, há séculos, acompanha a humanidade como um hábito lícito e socialmente aceitável, ligado à gratificação imediata, ao relaxamento e à facilitação da sociabilidade ${ }^{1}$. No entanto, estudos demonstram a íntima relação entre o consumo de bebidas alcoólicas e o risco de doenças, acidentes de trânsito, absenteísmo e acidentes de trabalho, envolvimento em brigas, violência intrafamiliar, violência sexual, criminalidade, dentre outros². A Organização Mundial de Saúde chama a atenção para as consequências do uso e abuso de álcool sobre a vida das pessoas e os impactos nas famílias e nações ${ }^{3}$.

Bebidas alcoólicas não são mercadorias comuns, e desta forma, não devem ser livremente comercializadas. Os problemas relacionados ao uso e ao abuso do álcool tem abrangência social ampla e transcende, em muito, o indivíduo que bebe. Assim, requerem políticas públicas abrangentes que disciplinem sua disponibilidade, acesso e consumo, no intuito de reduzir os riscos e os danos provenientes do seu uso e abuso ${ }^{1}$.

Além das doenças cardiovasculares, cirrose e neoplasias, o uso e o abuso de álcool estão fortemente associados ao uso de tabaco, de drogas ilícitas ${ }^{4}$ e a distúrbios psicossociais como depressão, transtorno de comportamento, perturbações e suicídios ${ }^{1}$. Episódios de violência também estão associados ao consumo de álcool. Em estudo de abrangência nacional, 38,1\% dos homens que agrediram as parceiras íntimas fizeram uso de álcool, entre as mulheres agressoras dos parceiros, 9,2\% tinham consumido bebida alcoólica ${ }^{5}$.

Acidentes de trânsito e lesões por causas externas têm sua magnitude ampliada por influência do consumo de bebidas alcoólicas ${ }^{6}$. Em Uberlândia (MG), 31,8\% dos pacientes que deram entrada em um hospital geral com lesões por causas externas apresentaram alcoolemia positiva, para as vitimas de agressão física o consumo de álcool foi confirmado em $57,1 \%$ dos pacientes ${ }^{7}$.

O perfil do consumo de álcool e as respectivas complicações estão relacionados ao volume ingerido e à frequência de uso. Dentre as principais complicações destacam-se o consumo abusivo e a dependência alcoólica. Por consumo abusivo ou uso nocivo entende-se a ingestão de álcool em dose elevada e frequência comum que pode trazer complicações físicas e psíquicas ao usuário. A dependência ou alcoolismo crônico caracteriza-se por estado de consumo regular que conduz a transtornos fisiológicos e comportamentais, com desejo poderoso de consumir, dificul- dade de controle, uso em diversos horários do dia e priorização do uso em detrimento de atividades e obrigações. Quando o uso não se faz de maneira regular o indivíduo entra em estado de abstinência ${ }^{8}$.

Sobre o perfil do uso abusivo e da dependência do uso de álcool, pesquisas demonstram uma maior associação com pessoas do sexo masculino e grupos etários mais jovens ${ }^{9,10}$. O elevado consumo entre a população mais jovem, sobretudo adolescentes, constitui-se em grave problema de saúde pública ${ }^{11}$.

As diversas variáveis relacionadas ao consumo abusivo e à dependência de bebidas alcoólicas apresentam associações bastante complexas. Fatores como escolaridade, renda e ocupação apresentam associações variadas com o uso de bebidas alcoólicas ${ }^{12}$. A determinação do padrão de consumo destas transcende questões biológicas, como sexo e idade, e são fortemente influenciadas pela estrutura social, fatores locais e regionais, e questões de ordem cultural, como as relativas aos grupos sociais de pertencimento.

Esse estudo teve como objetivo estimar a prevalência do consumo abusivo e da dependência de bebidas alcoólicas e os respectivos fatores associados em população urbana de um município do interior do Nordeste brasileiro.

\section{Métodos}

Estudo transversal com base populacional, realizado entre Janeiro e Março de 2010, que investigou aspectos relacionados ao consumo de bebidas alcoólicas em indivíduos maiores de 14 anos residentes na zona urbana de Jequié (BA).

O tamanho da amostra foi definido levando em consideração a prevalência de dependência de álcool para a Região Nordeste do Brasil em $2005,13,8 \%{ }^{13}$. Utilizou-se delta igual a 0,06 e nível de confiança de $95 \%$, para uma população infinita, efeito desenho igual a 2, e acréscimo de $10 \%$ para eventuais perdas e recusas em uma amostra sem reposição. Calculado no programa $\mathrm{R}$ versão 2.12.0 (R Project for Statistical Computing) apontou para uma amostra de aproximadamente 280 indivíduos.

A amostra foi definida em três estágios. Na primeira etapa, foram sorteados de maneira aleatória sistemática 15 setores censitários urbanos, de um total de 148. Para o segundo estágio, as quadras de cada setor foram dispostas sequencialmente, considerou-se a média de 250 domicílios por setor censitário, foi adotado intervalo 
sistemático de 12 domicílios. Os setores foram percorridos em suas quadras no sentido horário, considerados os domicílios do lado direito do logradouro pertencente à quadra. As quadras foram sucessivamente percorridas até atingir os 20 domicílios sorteados em cada setor censitário. Nos domicílios, para compor o terceiro estágio, todos os moradores maiores de 14 anos eram listados em ordem crescente de idade, então se identificava, na lista dos elegíveis, o indivíduo correspondente ao número aleatório previamente estabelecido na capa do formulário.

Os setores censitários foram percorridos e os domicílios foram visitados por uma equipe de seis entrevistadores treinados. Foi realizado estudo piloto, correspondente a $10 \%$ da amostra, em um setor censitário destinado exclusivamente para este fim. As entrevistas do estudo piloto não foram contabilizadas para a formação da amostra do estudo.

Para os indivíduos que não foram encontrados no domicílio no momento da primeira visita, foram agendadas até três tentativas de visita em horários diferentes.

Com relação ao consumo de bebidas alcoólicas, dois desfechos de interesse foram estabelecidos. O uso abusivo de álcool, definido por uma pontuação maior ou igual a 8 no escore obtido a partir das respostas ao Alcohol Use Disorders Identification Test (AUDIT) em sua versão brasilei$\mathrm{ra}^{14}$. A dependência de álcool foi identificada por uma pontuação igual ou superior a 2 obtida na aplicação da versão brasileira do questionário Cut-down, Annoyed by criticism, Guilty e Eyeopener (CAGE) ${ }^{8}$.

As variáveis independentes investigadas foram idade, gênero, ocupação (trabalhando, desempregado, dona de casa, estudante ou aposentado), cor da pele (autorreferida em branca, preta ou parda/indígena/amarela), crença religiosa (católica, evangélica, espírita ou nenhuma), renda familiar (até cinco salários mínimos ou mais que cinco salários mínimos), escolaridade (até oito anos de estudo, nove ou mais anos de estudo), estado civil (vivendo sem companheiro - solteiros, viúvos e divorciados; ou vivendo com companheiro - casados e em união estável) e tabagismo (fuma atualmente - sim ou não).

A análise estatística utilizou o modelo de Regressão Logística não condicional, no qual, para a obtenção do Odds Ratio bruto, foi empregada regressão logística simples para os desfechos de uso abusivo (AUDIT) e dependência de álcool (CAGE), usando diversos fatores como variáveis independentes. Também foi empregada regres- são múltipla para análise do Odds Ratio ajustado pelas variáveis independentes que apresentaram significância estatística $(\mathrm{p} \leq 0,05)$ na etapa anterior, para o desfecho uso abusivo (AUDIT).

Adotou-se p-valor $\leq 0,05$ como critério de significância. $\mathrm{O}$ teste de Wald foi utilizado para verificação de ajuste do modelo. Todos os procedimentos de análise foram realizados utilizando-se o programa computacional R versão 2.12.0 (R Project for Statistical Computing).

A pesquisa foi analisada e autorizada pelo Comitê de Ética em Pesquisa da Universidade Estadual do Sudoeste da Bahia. Foram observados todos os dispostos da ética em pesquisa com seres humanos, conforme preconizado pela Resolução 196/1996 ${ }^{15}$ do Conselho Nacional de Saúde.

\section{Resultados}

A amostra foi composta por 270 indivíduos distribuídos de maneira assimétrica entre os sexos: mulheres, 60,7\%; e homens, 39,3\%. A maior parte dos entrevistados, 60,7\%, mantinha-se trabalhando no período da entrevista e $2,6 \%$ estavam desempregados. Os residentes no município em estudo possuem baixo nível socioeconômico. Dos indivíduos que compuseram a amostra, mais da metade, $54,8 \%$, apresentaram oito ou menos anos de estudo e a maioria absoluta, 90,7\%, viviam em famílias com renda de cinco ou menos salários mínimos.

A prevalência do uso abusivo para bebidas alcoólicas, de acordo com o critério AUDIT $\geq 8$, foi de $18,5 \%$, sendo $34,9 \%$ para os homens e $7,9 \%$ para as mulheres. Para a dependência do consumo de álcool, CAGE $\geq 2$, a prevalência foi de $10,4 \%$, com $17 \%$ para os homens e $6,1 \%$ para as mulheres.

A Tabela 1 apresenta os resultados da prevalência do uso abusivo segundo as variáveis demográficas e sociais. Os fatores que se mostraram estatisticamente significante com o uso arriscado de álcool foram: faixa etária, gênero, crença religiosa e uso de tabaco. Observa-se relação inversamente proporcional entre o uso abusivo e a faixa etária. O grupo de adolescentes e adultos jovens $(\mathrm{OR}=5,28$; IC $95 \%=1,18$ a 23,69$)$ e as pessoas de meia idade $(\mathrm{OR}=4,29 ; \mathrm{IC} 95 \%=0,97$ a 18,99$)$ mantêm prevalência mais elevada quando comparado ao grupo de idosos. O sexo masculino mostra forte associação com o consumo prejudicial de álcool $(\mathrm{OR}=6,23 ; \mathrm{IC} 95 \%=3,11 \mathrm{a}$ 12,46). Para a religião, pessoas que professam crença evangélica mantêm prevalência significati- 
Tabela 1. Prevalência(\%) e Razões de chances (Odds ratio) do uso abusivo para bebidas alcoólicas (AUDIT $\geq 8$ ) segundo variáveis sócio demográficas e sociais. Regressão simples.

\begin{tabular}{|c|c|c|c|c|c|c|}
\hline Variável & $\mathbf{n}$ & $\begin{array}{c}\text { Prevalência } \\
(\%)\end{array}$ & IC (95\%) & $\begin{array}{l}\text { Odds } \\
\text { ratio }\end{array}$ & IC $(95 \%)$ & $\mathrm{p}$ \\
\hline \multicolumn{7}{|l|}{ Faixa etária (anos) } \\
\hline $14-29$ & 97 & 22,7 & 17,7 a 27,7 & 5,28 & 1,18 a 23,69 & 0,030 \\
\hline $30-59$ & 135 & 19,3 & 14,6 a 24,0 & 4,29 & 0,97 a 18,99 & 0,055 \\
\hline$\geq 60$ & 38 & 5,3 & 2,6 a 8,0 & 1 & & \\
\hline \multicolumn{7}{|l|}{ Gênero } \\
\hline Masculino & 106 & 34,9 & 29,2 a 40,6 & 6,23 & 3,11 a 12,46 & $<0,001$ \\
\hline Feminino & 164 & 7,9 & 4,7 a 11,1 & 1 & & \\
\hline \multicolumn{7}{|l|}{ Ocupação } \\
\hline Desempregado & 7 & 28,6 & 23,2 a 34,0 & 1,49 & 0,28 a 7,99 & 0,645 \\
\hline Dona de casa & 38 & 13,2 & 9,2 a 17,2 & 0,56 & 0,21 a 1,55 & 0,266 \\
\hline Estudante & 39 & 17,9 & 13,3 a 22,5 & 0,81 & 0,33 a 2,00 & 0,651 \\
\hline Aposentado & 21 & 4,8 & 2,2 a 7,4 & 0,19 & 0,02 a 1,43 & 0,106 \\
\hline Trabalhando & 164 & 21,3 & 16,4 a 26,2 & 1 & & \\
\hline \multicolumn{7}{|l|}{ Cor da pele } \\
\hline Preta & 53 & 13,2 & 9,2 a 17,2 & 0,87 & 0,28 a 2,69 & 0,808 \\
\hline Parda & 170 & 21,2 & 16,3 a 26,1 & 1,54 & 0,64 a 3,71 & 0,342 \\
\hline Branca & 47 & 14,9 & 10,7 a 19,1 & 1 & & \\
\hline \multicolumn{7}{|l|}{ Crença religiosa } \\
\hline Nenhuma & 40 & 35,0 & 29,3 a 40,7 & 1,77 & 0,83 a 3,75 & 0,137 \\
\hline Espírita & 2 & - & - & - & - & \\
\hline Evangélica & 78 & 1,3 & 0 a 2,7 & 0,04 & 0,01 a 0,32 & 0,002 \\
\hline Católica & 150 & 23,3 & 18,3 a 28,3 & 1 & & \\
\hline \multicolumn{7}{|l|}{ Renda Familiar } \\
\hline$\leq 5$ salários mínimos & 245 & 18,4 & 13,7 a 23,1 & 1 & & \\
\hline 6 ou mais salários mínimos & 20 & 25,0 & 19,8 a 30,2 & 1,48 & 0,51 a 4,29 & 0,468 \\
\hline \multicolumn{7}{|l|}{ Escolaridade } \\
\hline$\leq 8$ anos de estudo & 148 & 18,2 & 13,6 a 22,8 & 1 & & \\
\hline 9 ou mais anos de estudo & 122 & 18,9 & 14,2 a 23,6 & 1,04 & 0,56 a 1,93 & 0,898 \\
\hline \multicolumn{7}{|l|}{ Estado Civil } \\
\hline Vivendo sem companheiro & 129 & 20,2 & 15,3 a 25,1 & 1,21 & 0,65 a 2,25 & 0,553 \\
\hline Vivendo com companheiro & 133 & 17,3 & 12,7 a 21,9 & 1 & & \\
\hline \multicolumn{7}{|l|}{ Tabagismo } \\
\hline Sim & 30 & 36,7 & 31,0 a 42,4 & 2,97 & 1,31 a 6,73 & 0,009 \\
\hline Não & 240 & 16,3 & 11,9 a 20,7 & 1 & & \\
\hline
\end{tabular}

Fonte: Dados da pesquisa. Jequié (BA), 2010.

vamente menor $(\mathrm{OR}=0,04 ; \mathrm{IC} 95 \%=0,01$ a 0,32$)$ quando comparados aos católicos. Os tabagistas apresentam maior chance $(\mathrm{OR}=2,97$; IC $95 \%=$ 1,31 a 6,73) de consumo abusivo de álcool.

Para a dependência de álcool foi encontrada associação estatística com as variáveis gênero, religião e tabagismo (Tabela 2). Ser do sexo masculino ( $\mathrm{OR}=3,15$; IC 95\% = 1,39 a 7,13) e fazer uso de tabaco (OR = 3,98; IC 95\% = 1,57 a 10,09) mostraram-se associados positivamente com a dependência de álcool. A crença evangélica ( $O R$ $=0,19 ;$ IC $95 \%=0,04$ a 0,85$)$ apresenta associação negativa quando comparados com os de religião católica.
No modelo de regressão logística multivariada para o consumo abusivo de álcool, mantiveram-se associação estatística significante a faixa etária de 14 aos 29 anos, o sexo masculino e a religião evangélica (Tabela 3 ). Observa-se que a faixa etária de 30 aos 59 anos e os tabagistas não conservaram associação estatística na análise ajustada. Para os fatores que se mantiveram associados, não se observou grande variação do efeito quando comparados à análise bruta. O grupo de idade mais jovem apresentou $\mathrm{OR}=5,41$ (IC $95 \%=1,11$ a 26,30 ), o sexo masculino $\mathrm{OR}=5,56$ (IC 95\% = 2,66 a 11,60) e os que professam crença evangélica OR =0,04 (IC 95\%=0,01 a 0,31). 
Tabela 2. Prevalência (\%) da dependência de bebidas alcoólicas (CAGE $\geq 2$ ) e Razões de chances (Odds ratio) segundo variáveis sociodemográficas e sociais. Regressão simples.

\begin{tabular}{|c|c|c|c|c|c|c|}
\hline Variável & $\mathbf{n}$ & Prevalência (\%) & IC (95\%) & Odds ratio & IC $(95 \%)$ & $\mathbf{p}$ \\
\hline \multicolumn{7}{|l|}{ Faixa etária (anos) } \\
\hline $14-29$ & 97 & 12,4 & 8,5 a 16,3 & 1,65 & 0,44 a 6,20 & 0,460 \\
\hline $30-59$ & 135 & 9,6 & 6,1 a 13,1 & 1,24 & 0,34 a 4,61 & 0,745 \\
\hline$\geq 60$ & 38 & 7,9 & 4,7 a 11,1 & 1 & & \\
\hline \multicolumn{7}{|l|}{ Gênero } \\
\hline Masculino & 106 & 17,0 & 12,5 a 21,5 & 3,15 & 1,39 a 7,13 & 0,006 \\
\hline Feminino & 164 & 6,1 & 3,2 a 9,0 & 1 & & \\
\hline \multicolumn{7}{|l|}{ Ocupação } \\
\hline Desempregado & 7 & 14,3 & 10,1 a 18,5 & 1,45 & 0,17 a 12,78 & 0,737 \\
\hline Dona de casa & 38 & 13,2 & 9,2 a 17,2 & 1,32 & 0,45 a 3,83 & 0,611 \\
\hline Estudante & 39 & 12,8 & 8,8 a 16,8 & 1,28 & 0,44 a 3,71 & 0,649 \\
\hline Aposentado & 21 & - & - & - & - & - \\
\hline Trabalhando & 164 & 10,4 & 6,8 a 14,0 & 1 & & \\
\hline \multicolumn{7}{|l|}{ Cor da pele } \\
\hline Preta & 53 & 5,7 & 2,9 a 8,5 & 0,88 & 0,17 a 4,59 & 0,879 \\
\hline Parda & 170 & 12,9 & 8,9 a 16,9 & 2,18 & 0,62 a 7,63 & 0,223 \\
\hline Branca & 47 & 6,4 & 3,5 a 9,3 & 1 & & \\
\hline \multicolumn{7}{|l|}{ Crença religiosa } \\
\hline Nenhuma & 40 & 20,0 & 15,2 a 24,8 & 1,83 & 0,73 a 4,59 & 0,196 \\
\hline Espírita & 2 & - & - & - & - & , \\
\hline Evangélica & 78 & 2,6 & 0,7 a 4,5 & 0,19 & 0,04 a 0,85 & 0,030 \\
\hline Católica & 150 & 12,0 & 8,1 a 15,9 & 1 & & \\
\hline \multicolumn{7}{|l|}{ Renda Familiar } \\
\hline$\leq 5$ salários mínimos & 245 & 10,6 & 6,9 a 14,3 & 1 & & \\
\hline 6 ou mais salários mínimos & 20 & 10,0 & 6,4 a 13,6 & 0,94 & 0,21 a 4,26 & 0,932 \\
\hline \multicolumn{7}{|l|}{ Escolaridade } \\
\hline$\leq 8$ anos de estudo & 148 & 13,5 & 9,4 a 17,6 & 1 & & \\
\hline 9 ou mais anos de estudo & 122 & 6,6 & 3,6 a 9,6 & 0,45 & 0,19 a 1,06 & 0,067 \\
\hline \multicolumn{7}{|l|}{ Estado Civil } \\
\hline Vivendo sem companheiro & 129 & 13,2 & 9,1 a 17,3 & 1,68 & 0,76 a 3,75 & 0,202 \\
\hline Vivendo com companheiro & 133 & 8,3 & 5,0 a 11,6 & 1 & & \\
\hline \multicolumn{7}{|l|}{ Tabagismo } \\
\hline Sim & 30 & 26,7 & 21,4 a 32,0 & 3,98 & 1,57 a 10,09 & 0,004 \\
\hline Não & 240 & 8,3 & 5,0 a 11,6 & 1 & & \\
\hline
\end{tabular}

Fonte: Dados da pesquisa. Jequié (BA), 2010.

\section{Discussão}

A prevalência do uso abusivo de álcool encontrada em Jequié (BA) (18,5\%) foi consideravelmente maior do que a de Campinas (SP ${ }^{16}, 8,4 \%$. No que tange a prevalência da dependência de álcool, o resultado da presente investigação $(10,4 \%)$ guarda semelhança com os achados de estudos com abrangência nacional. Laranjeira et al. ${ }^{17}$ estimaram uma prevalência de $9 \%$ da dependência de álcool em adultos no país. Em outro estudo, também de porte nacional, a prevalência estimada é de $12,3 \%$, para a população acima de 12 anos $^{13}$.
Neste estudo, verificou-se que os jovens apresentam maior chance de uso abusivo $(\mathrm{OR}=5,28)$ e dependência $(\mathrm{OR}=1,65)$ quando comparados aos demais grupos etários. A associação entre uso abusivo e faixa etária se manteve após análise ajustada.

Desta forma, é possível afirmar que o grupo de menor idade, independente das demais variáveis, apresenta cinco vezes mais chance de uso abusivo de bebidas alcoólicas do que os idosos. Os dados obtidos se coadunam com os resultados de Campinas $^{16}$, ao passo que na cidade de Salvador $(\mathrm{BA})^{18}$, foram encontradas maiores chances de uso abusivo nas faixas etárias de maior idade. 
Tabela 3. Prevalência (\%) e Razões de chances ajustada (Odds ratio) do uso abusivo para bebidas alcoólicas (AUDIT $\geq 8$ ) segundo variáveis sócio demográficas e sociais. Regressão múltipla.

\begin{tabular}{|c|c|c|c|c|c|c|}
\hline Variável & $\mathbf{N}$ & Prevalência (\%) & IC (95\%) & Odds ratio & IC $(95 \%)$ & $\mathbf{P}$ \\
\hline \multicolumn{7}{|c|}{ Faixa etária (anos) } \\
\hline $14-29$ & 97 & 22,7 & 17,7 a 27,7 & 5,41 & 1,11 a 26,30 & 0,036 \\
\hline $30-59$ & 135 & 19,3 & 14,6 a 24,0 & 3,90 & 0,82 a 18,45 & 0,087 \\
\hline$\geq 60$ & 38 & 5,3 & 2,6 a 8,0 & 1 & & \\
\hline \multicolumn{7}{|l|}{ Gênero } \\
\hline Masculino & 106 & 34,9 & 29,2 a 40,6 & 5,56 & 2,66 a 11,60 & $<0,001$ \\
\hline Feminino & 164 & 7,9 & 4,7 a 11,1 & 1 & & \\
\hline \multicolumn{7}{|l|}{ Crença religiosa } \\
\hline Nenhuma & 40 & 35,0 & 29,3 a 40,7 & 1,18 & 0,52 a 2,70 & 0,691 \\
\hline Espírita & 2 & - & - & - & - & \\
\hline Evangélica & 78 & 1,3 & 0 a 2,7 & 0,04 & 0,01 a 0,31 & 0,002 \\
\hline Católica & 150 & 23,3 & 18,3 a 28,3 & 1 & & \\
\hline \multicolumn{7}{|l|}{ Tabagismo } \\
\hline Sim & 30 & 36,7 & 31,0 a 42,4 & 1,68 & 0,66 a 4,24 & 0,274 \\
\hline Não & 240 & 16,3 & 11,9 a 20,7 & 1 & & \\
\hline
\end{tabular}

Fonte: Dados da pesquisa. Jequié (BA), 2010.

A despeito das diferenças encontradas, o elevado consumo entre a população mais jovem é fator preocupante, pois além da vulnerabilidade à violência, aos acidentes de trânsito e ao uso de outras drogas ilícitas, os costumes nas idades mais jovens podem moldar comportamentos futuros e as consequências do uso e abuso de álcool se estenderem para outros ciclos da vida ${ }^{19}$.

Dentre os fatores relacionados ao elevado consumo de álcool na população mais jovem, as relações familiares conflituosas apresentam-se como fator diretamente associado a esse hábito e a doutrina religiosa consitui-se em varável inversamente relacionada ${ }^{20,21}$. No entanto, estudos evidenciam a grande influência da propaganda de bebidas alcóolicas na indução do consumo precoce desses produtos ${ }^{22}$.

A propaganda de bebidas alcoólicas no Brasil, de qualidade e criatividade destacadas ${ }^{20}$, busca associar o seu consumo a situações de prazer, de alegria e de pertencimento a grupos sociais atrativos. Os ambientes que são apresentados nas peças publicitárias estão sempre permeados de belas mulheres, homens realizados e situações de descontração. Essa imagem utilizada denota grande poder atrativo, especialmente em adolescentes e adultos jovens que, em busca dessa situação ideal, se inclinam ao consumo precoce e elevado desses produtos.

Estudos demonstram que o forte apelo do marketing de bebidas é meticulosamente pensado e direcionado para o público jovem, de modo a formar novos consumidores ${ }^{22}$. Assim, o excesso de exposição às propagandas tende a aumentar o consumo neste público. Como agravante, o Brasil ainda apresenta frágil regulação da propaganda e do comércio de bebidas alcóolicas. Esta situação se coloca na contramão da responsabilidade esperada do Estado e da sociedade, visto que as bebidas alcoólicas não são mercadorias comuns, não devem estar restritas apenas às leis de mercado e, portanto, demandam forte controle para o seu comércio e divulgação ${ }^{1}$.

Associado a isso, apesar de ser proibida a comercialização de bebidas alcoólicas para menores de 18 anos no Brasil, é evidente que a parca fiscalização e o fácil acesso e disponibilidade de bebidas para menores também contribuem para o seu elevado consumo ${ }^{23}$.

Entre a população de maior idade, os resultados encontrados corroboram os achados de outros estudos que evidenciam menores prevalências para o consumo elevado de álcool nessa faixa etária ${ }^{16,24}$. No entanto, faz-se mister monitorar os padrões de consumo entre as pessoas de idade mais avançada no intuito de identificar possíveis implicações e prejuízos à saúde. Em uma coorte acompanhada durante 11 anos foi encontrada incrementos nos riscos potenciais de consumo abusivo de bebidas alcoólicas entre as faixas etárias a partir de 42 anos $^{2}$.

A variável gênero mostrou-se associada tanto com o uso abusivo quanto com a dependência de álcool. Para o uso abusivo, o gênero masculino 
manteve associação significante na análise bruta, que se manteve na análise ajustada. O maior consumo e a dependência de álcool entre os homens é uma realidade presente em diversos países ${ }^{2,12,17}$. A cultura de dominação masculina e a associação do álcool aos momentos de lazer, relaxamento e descontração desempenham forte influência para o estabelecimento dessa realidade.

No entanto, neste estudo, chama atenção a elevada prevalência do uso abusivo entre os homens (34,9\%), enquanto em Pelotas (RS) foi de $27,2 \%{ }^{25}$, em Campinas (SP) foi de $13,1 \%{ }^{16}$ e em Salvador (BA) foi de $12,4 \%{ }^{18}$.

Para o gênero feminino, a prevalência do consumo abusivo de álcool foi de 7,9\% e da dependência, 6,1\%. Esses resultados são levemente superiores ao encontrados em outros ${ }^{16,25}$ e se mantém próximo do valor estimado da prevalência nacional, 6,9\%, para as mulheres ${ }^{13}$.

Embora o consumo entre as mulheres seja consideravelmente menor do que entre os homens, Carlini et al. ${ }^{13}$ apontam uma tendência de crescimento do uso e abuso de álcool entre as mulheres. Isto porque, à medida que os papéis das mulheres se tornam semelhantes aos dos homens, o padrão de consumo de álcool também tende a modificar-se $\mathrm{e}^{26}$.

Mesmo que as prevalências no gênero masculino sejam mais elevadas, é preocupante a tendência de redução das diferenças. As mulheres são mais susceptíveis aos prejuízos por bebidas alcoólicas do que os homens, tanto para fatores biológicos ${ }^{27}$, quanto para a vulnerabilidade à violência associada ao seu consumo ${ }^{5}$.

Com relação à crença religiosa, as prevalências de uso abusivo e da dependência de álcool entre os evangélicos foram consideravelmente menores quando comparadas aos de religião católica. Aqueles sem filiação religiosa não mostraram associação significante. O modelo de regressão ajustada evidenciou a manutenção da associação inversa da dependência de álcool com o grupo dos evangélicos. Esses resultados guardam semelhança com estudo realizado entre adolescentes brasileiros no qual também foi encontrada uma menor prevalência de uso abusivo de álcool entre os que mantêm vinculação a grupos religiosos ${ }^{20}$.

Uma razão explicativa para esse fenômeno, pode advir da forte indução exercida pelas igrejas evagélicas sobre o comportamento social dos seus membros. Especialmente nas cidades do interior, as religiões evangélicas porcuram distinguir o seu público do restante da população ao atribuir aos fiéis a busca por uma conduta de retidão com afastamento do uso de alcóol, de tabaco e de drogas ilícitas.

No que se refere ao tabagismo, o presente estudo encontrou prevalência de $11,1 \%$ de fumantes, proporção muito parecida aos 10,1\% encontrados em levantamento nacional ${ }^{13}$. Foi possível observar que o hábito de fumar manteve-se associado ao uso abusivo e à dependência de álcool. $\mathrm{Na}$ análise ajustada o consumo de tabaco perdeu significância em sua associação com o costume etílico.

A associação entre o tabagismo e consumo abusivo e/ou dependência alcoólica é uma condição clássica ${ }^{4}$, marcada pela potenciação de um hábito sobre o outro. Observa-se farta documentação desta associação nos estudos epidemiológicos $^{24,28}$. Além da associação com o tabaco, o consumo de álcool apresenta-se também associado a outras drogas ilícitas ${ }^{4,16,29}$.

Os resultados da presente investigação não demonstraram associação significante entre o consumo elevado de álcool e as variáveis ocupação, cor da pele, renda familiar, escolaridade e estado civil. Para algumas dessas variáveis, os achados publicados na literatura mostram também uma não associação com uso excessivo de álcool, ao passo que para outras, os resultados são inconclusivos no que tange a natureza dessa associação.

Em diversos estudos brasileiros também não foi encontrada associação entre o consumo abusivo de bebidas alcoólicas e a variável estado civil ${ }^{16,18,25}$. A cor da pele não foi significante nos achados de Almeida-Filho et al. ${ }^{18}$. E a ocupação também não se mostrou associada à dependência de álcool no estudo realizado em Bebedouro (SP) ${ }^{24}$.

As variáveis renda e escolaridade apresentam situações ainda mais peculiares em sua associação com consumo abusivo e/ou com a dependência de álcool. Isto porque, a literatura da área evidencia controvérsias entre uso elevado de bebidas alcoólicas e os diferentes estratos populacionais.

Não obstante a associação não significativa entre a renda e o consumo elevado de álcool em Jequié (BA), a prevalência do abuso e dependência de álcool se mostra associada tanto com grupos de menor renda ${ }^{24}$, quanto aos segmentos mais abastados ${ }^{16,18}$.

De maneira análoga, também não está clara na literatura a associação entre o nível educacional e o consumo arriscado ou dependência de bebidas alcoólicas.

Os estratos com níveis educacionais mais elevados tendem a conferir comportamento saudável em se tratando dos padrões de consumo 
de cigarro e bebidas alcoólicas ${ }^{12}$. No entanto, os estudos realizados no Brasil demonstram realidades diferentes para associação entre consumo de álcool e escolaridade. Algumas pesquisas ${ }^{16,25}$ demonstram maiores prevalências nos grupos de menor escolaridade, ao passo que em ou$\operatorname{tras}^{18,24,30}$ são observadas associação com os estratos de maior escolaridade.

Essa aparente contradição relativa à associação entre esses fatores é sugestiva de que as variáveis relacionadas ao consumo elevado de álcool sofrem diversas influências e se conjugam entre si de maneira bastante complexa.

Há evidências também, de que o contexto em que se bebe, influencia o padrão de consumo e a iniciação precoce de novos bebedores ${ }^{1,31}$. Neste sentido, as elevadas prevalências do uso abusivo e da dependência podem ser influenciadas, no caso específico deste estudo, pelas escassas opções de lazer que não tenham apelo ao consumo de bebidas alcoólicas.

O município de Jequié (BA) caracteriza-se como de médio porte, economia pouco desenvolvida e parcos equipamentos públicos destinados ao lazer, ao esporte e à cultura. Desta forma, o apelo ao consumo de álcool ganha eco na população carente de opções de divertimento, especialmente a mais jovem. Assim, como alternativa para diminuição do consumo de álcool, devem ser pensadas não apenas políticas regulatórias destinadas à inibição da propaganda e do comércio de bebidas, mas, sobretudo, políticas públicas intersetoriais, com capacidade ampliar as perspectivas de vida da população.

Por fim, devem ser ponderadas as restrições metodológicas do presente estudo. Como a pes- quisa foi do tipo transversal e utilizou instrumento de inquérito recordatório, existem limitações inerentes à natureza do método, a exemplo dos possíveis vieses dos inquéritos de autodeclaração. Além da possibilidade do viés de memória, posições socioeconômicas muitas vezes são negadas por interferência de preconceitos e discriminações, o que leva algumas pessoas a não se reconhecerem ou terem vergonha de se declarar integrantes de determinados grupos sociais.

\section{Colaboradores}

LN Ferreira participou da concepção do estudo, coordenação da coleta de dados, análise dos dados e redação do texto científico. JP Bispo Júnior colaborou com a análise dos dados e redação do texto final. ZN Sales foi a orientadora e participou da redação do texto final. CA Casotti colaborou com a orientação do estudo e redação do texto final. ACR Braga Júnior colaborou com a análise estatística e a revisão do texto final.

\section{Agradecimentos}

À Universidade Estadual do Sudoeste da Bahia pelo financiamento da pesquisa. 


\section{Referências}

1. Barbor TF, Caetano R, Casswell S, Edwards G, Giesbrect N, Graham K, Grube J, Gruenewald P, Hill L, Holder H, Homel R, Osterberg E, Rehm J, Room R, Rossow I. Alcohol : no ordinary commodity : research and public policy. 2a Edição. Oxford: Oxford University Press; 2010.

2. Ilomäki J, Korhonen MJ, Lavikainen P, Lipton R, Enlund H, Kauhanen J. Changes in alcohol consumption and drinking patterns during 11 years of follow-up among ageing men: the FinDrink study. Eur J Public Health 2010; 20(2):133-138.

3. World Health Organization (WHO). Global status report on alcohol and health. Geneva: WHO; 2011.

4. Iglesias V, Cavada G, Silva C, Cáceres D. Consumo precoz de tabaco y alcohol como factores modificadores del riesgo de uso de marihuana. Rev Saude Publica 2007; 41(4):517-522.

5. Zaleski M, Pinsky I, Laranjeira R, Ramisetty-Mikler S, Caetano R. Violência entre parceiros íntimos e consumo de álcool. Rev Saude Publica 2010; 44(1):53-59.

6. Mascarenhas MDM, Malta DC, Silva MMA, Carvalho CG, Monteiro RA, Morais Neto OL. Consumo de álcool entre vítimas de acidentes e violências atendidas em serviços de emergência no Brasil, 2006 e 2007. Cien Saude Colet 2009; 14(5):1789-1796.

7. Freitas EAM, Mendes ID, Oliveira LCM. Ingestão alcoólica em vítimas de causas externas atendidas em um hospital geral universitário. Rev Saude Publica 2008; 42(5):813-821.

8. Castells MA, Furlanetto LM. Validity of the CAGE questionnaire for screening alcohol-dependent inpatients on hospital wards. Rev Bras Psiquiatr 2005; 27(1):54-57.

9. Ferreira LN, Sales ZN, Casotti CA, Bispo Júnior JP, Braga Júnior ACR. Perfil do consumo de bebidas alcoólicas e fatores associados em um município do Nordeste do Brasil. Cad Saude Publica 2011; 27(8):1473-1486.

10. Galduróz JCF, Carlini EA. Use of alcohol among the inhabitants of the 107 largest cities in Brazil 2001. Braz J Med Biol Res 2007; 40(3):367-375.

11. Reichenheim ME, Souza ER, Moraes CL, Mello Jorge MHP, Silva CMFP, Minayo MCS. Violence and injuries in Brazil: the effect, progress made, and challenges ahead. Lancet 2011; 6736(11):75-89

12. Johnson W, Kyvik KO, Mortensen EL, Skytthe A, Batty GD, Deary IJ. Does Education Confer a Culture of Healthy Behavior? Smoking and Drinking Patterns in Danish Twins. Am J Epidemiol 2011; 173(1):55-63.

13. Carlini EA, Galduróz JCF, Noto AR, Fonseca AM, Carlini CM, Oliveira LG, Nappo SA, Moura YG, Sanchez ZVDM. II Levantamento Domiciliar sobre o uso de Drogas Psicotrópicas no Brasil: Estudo envolvendo as 108 maiores cidades do país 2005. Brasília: Secretaria Nacional Antidrogas, CEBRID, UNIFESP; 2007.

14. Lima CT, Freire ACC, Silva APB, Teixeira RM, Farrell $\mathrm{M}$, Prince $\mathrm{M}$. Concurrent and Construct Validity of the AUDIT in an Urban Brazilian Sample. Alcohol Alcohol 2005; 40(6):584-589.
15. Brasil. Ministério da Saúde (MS). Conselho Nacional de Saúde. Resolução no 196 de 10 de outubro de 1996. Diretrizes e Normas Regulamentadoras de Pesquisas Envolvendo Seres Humanos. Diário Oficial da União 1996; 16 out.

16. Barros MBA, Botega NJ, Dalgalarrondo P, MarínLeón L, Oliveira HB. Prevalence of alcohol abuse and associated factors in a population-based study. Rev Saude Publica 2007; 41(4):502-509.

17. Laranjeira R, Pinsky I, Sanches M, Zaleski M, Caetano R. Alcohol use patterns among Brazilian adults. Rev Bras Psiquiatr 2010; 32(3):231-241.

18. Almeida-Filho N, Lessa I, Magalhães L, Araújo MJ, Aquino E, Kawachi I, James SA. Alcohol drinking patterns by gender, ethnicity, and social class in Bahia, Brazil. Rev Saude Publica 2004; 38(1):45-54.

19. Vieira DL, Ribeiro M, Laranjeira R. Evidence of association between early alcohol use and risk of later problems. Rev Bras Psiquiatr 2007; 29(3):222-227.

20. Galduróz JCF, Sanchez ZM, Opaleye ES, Noto AR, Fonseca AM, Gomes PLS, Carlini EA. Fatores associados ao uso pesado de álcool entre estudantes das capitais brasileiras. Rev Saude Publica 2010; 44(2): 267-273.

21. Noal RB, Menezes AMB, Araújo CL, Hallal PC. Experimental use of alcohol in early adolescence: the 11-year follow-up of the 1993 Pelotas (Brazil) birth cohort study. Cad Saude Publica 2010; 26(10): 1937-1944.

22. Morgenstern M, Isensee B, Sargent JD, Hanewinkel R. Exposure to alcohol advertising and teen drinking. Prev Med 2011; 52(2):146-151.

23. Romano M, Duailibi S, Pinsky I, Laranjeira R. Pesquisa de compra de bebidas alcoólicas por adolescentes em duas cidades do Estado de São Paulo. Rev Saude Publica 2007; 41(4):495-501.

24. Vargas D, Oliveira MAF, Araújo EC. Prevalência de dependência alcoólica em serviços de atenção primária à saúde de Bebedouro, São Paulo, Brasil. Cad Saude Publica 2009; 25(8):1711-1720.

25. Costa JSD, Silveira MF, Gazalle FK, Oliveira SS, Hallal PC, Menezes AMB, Gigante DP, Olinto MTA, Macedo S. Consumo abusivo de álcool e fatores associados: estudo de base populacional. Rev Saude Publica 2004; 38(2):284-291.

26. Kerr-Corrêa F, Tucci AM, Hegedus AM, Trinca LA, Oliveira JB, Floripes TMF, Kerr LR. Drinking patterns between men and women in two distinct Brazilian communities. Rev Bras Psiquiatr 2008; 30(3): 235-242.

27. Ceylan-Isik AF, McBride SM, Ren J. Sex difference in alcoholism: Who is at a greater risk for development of alcoholic complication? Life Sci 2010; 87(56):133-138

28. Guimarães VV, Florindo AA, Stopa SR, César CLG, Barros MBA, Carandina L, Goldbaum M. Consumo abusivo e dependência de álcool em população adulta no Estado de São Paulo, Brasil. Rev Bras Epidemiol 2010; 13(2):314-325.

29. Fonseca AM, Galduróz JCF, Noto AR, Carlini ELA. Comparison between two household surveys on psychotropic drug use in Brazil: 2001 and 2004. Cien Saude Colet 2010; 15(3):663-670. 
30. Bortoluzzi MC, Traebert J, Loguercio A, Kehrig RT. Prevalência e perfil dos usuários de álcool de população adulta em cidade do sul do Brasil. Cien Saude Colet 2010; 15(3):679-685.

31. Souza MLP, Deslandes SF, Garnelo L. Modos de vida e modos de beber de jovens indígenas em um contexto de transformações. Cien Saude Colet 2010; 15(3):709-716.

Artigo apresentado em 26/05/2012

Aprovado em 19/07/2012

Versão final apresentada em 30/07/2012 